\title{
Effects of electroacupuncture on spinal $\alpha$-amino-3-hydroxy-5-methyl-4-isoxazole propionic acid receptor in rats injected with complete Freund's adjuvant
}

\author{
YOUNG-SEOK LEE ${ }^{1}$, JUN-HYUK LEE ${ }^{2}$, IN-SEON LEE ${ }^{1}$ and BYUNG-TAE CHOI ${ }^{3}$ \\ ${ }^{1}$ Department of Rehabilitation, College of Oriental Medicine, Dong-eui University, Busanjn-gu, Busan 614-052; \\ ${ }^{2}$ Chemistry and Biotechnology Examination Bureau, Korean Intellectual Property Office, Seo-gu, Daejeon 302-701; \\ ${ }^{3}$ Division of Meridian and Structural Medicine, School of Korean Medicine, Pusan National University, \\ Yangsan, Gyeongnam 626-870, Republic of Korea
}

Received February 15, 2013; Accepted June 17, 2013

DOI: $10.3892 / \mathrm{mmr} .2013 .1633$

\begin{abstract}
The effects of electroacupuncture (EA) on spinal $\alpha$-amino-3-hydroxy-5-methyl-4-isoxazole-propionic acid (AMPA) receptor subunits in male rats injected with complete Freund's adjuvant (CFA) were investigated. Bilateral EA stimulation ( $2 \mathrm{~Hz}, 1 \mathrm{~mA}$ ) was administered by needle insertion for 30 min once daily at acupoints corresponding to Zusanli and Sanyinjiao, and the thermal thresholds were measured. To examine the changes in the AMPA receptor subunits, the L4-5 segments of the spinal cord were analyzed by qPCR, western blot analysis and immunohistochemistry. The CFA-induced thermal sensitivity of the rat hind paw was significantly inhibited by EA stimulation from day 3 following CFA injection. On day 5 following CFA injection, there were no significant changes in the expression of the AMPA receptor GluR1 subunit in the CFA-injected rats with or without EA stimulation, compared with the control rats. However, the expression of the GluR2 subunit was significantly decreased by CFA treatment. Western blot analysis revealed that the expression of the phosphorylated GluR1 subunit in the control rats was not significantly different compared with that in the CFA-injected rats with and without EA stimulation. However, phosphorylation of the ipsilateral GluR2 subunit was significantly increased in the CFA-injected rats, and this activation was prevented by EA stimulation. Immunohistochemical analysis revealed a greater expression of phospho-GluR2 following CFA injection, which was inhibited by EA stimulation. These results suggested that phosphorylation of the AMPA receptor, particularly the GluR2 subunit, may be important in EA analgesia of CFA-induced inflammation.
\end{abstract}

Correspondence to: Dr Byung-Tae Choi, Division of Meridian and Structural Medicine, School of Korean Medicine, Pusan National University, Yangsan, Gyeongnam 626-870, Republic of Korea E-mail: choibt@pusan.ac.kr

Key words: electroacupuncture, hyperalgesia, AMPA receptor, CFA

\section{Introduction}

Glutamate, the primary rapid excitatory neurotransmitter, elicits postsynaptic effects by activating three main classes of ionotropic glutamate receptor, termed according to the selective agonists, $N$-methyl-D-aspartate (NMDA), $\alpha$-amino3-hydroxy-5-methyl-4-isoxazole-propionic acid (AMPA) and kainate (KA) (1). The NMDA receptor contributes to excitatory synaptic transmission within the spinal cord and is an important target in inflammatory pain $(2,3)$. The AMPA receptor also plays a critical role in the dorsal horn of the spinal cord in the processing of nociceptive information that is involved in persistent inflammatory pain (4-6).

The spinal AMPA receptor is a heterotetrameric cation channel composed of four GluR1-R4 subunits consisting of homo- and heteromultimers $(1,4,6)$. All four subunits are expressed within the spinal dorsal horn, with the GluR1 and GluR2 subunits being the most abundant in the superficial dorsal horn $(7,8)$. Peripheral inflammation induced by complete Freund's adjuvant (CFA) leads to GluR1 membrane insertion and GluR2 internalization in dorsal horn neurons (9). The phosphorylation of serine residues of the AMPA receptor is one of the key mechanisms in regulating channel properties $(2,8)$.

Electroacupuncture (EA) is widely used in clinical and basic research in Korea. The AMPA receptor may be involved in the induction of EA analgesia in the spinal dorsal horn of healthy animals (10); thus, any changes in the constituent subunits may affect EA analgesia. However, it is unclear whether EA analgesia is associated with selective changes in AMPA receptor subunits at the transcriptional or the translational level. The present study investigated the possible involvement of the AMPA receptor and the phosphorylation of its constituent serine residues on EA analgesia in chronic inflammatory pain.

\section{Subjects and methods}

Animals. Male Sprague-Dawley rats, (mean weight, $120 \mathrm{~g}$ ), were obtained from Dooyeol Biotech Co. (Seoul, Korea). The rats were housed at $22^{\circ} \mathrm{C}$ with an alternating 12-h dark-light 
cycle, were fed a commercial diet and water was provided ad libitum from 2 weeks prior to and throughout the study. All experiments conformed to guidelines approved by the Animal Care and Use Committee at the Pusan National University (Yangsan, Korea).

CFA injection and EA simulation. Rats were injected subcutaneously with $100 \mu \mathrm{l}$ CFA (Sigma-Aldrich, St. Louis, MO, USA) in the plantar surface of the left hindpaw. For the EA stimulation, two $0.25 \mathrm{~mm}$-diameter needles were inserted in each hind leg at acupoints corresponding to Zusanli (ST36) and Sanyinjiao (SP6) as described in a previous study (10). The needles were connected to a model SM-60 electric stimulator (Saechang, Seoul, Korea). EA stimulation (2 Hz, $1.0 \mathrm{~mA}$ ) was initiated immediately following injection with CFA and lasted for $30 \mathrm{~min}$. This process was repeated daily for the remainder of the experiment. Rats in the control group were injected with $100 \mu \mathrm{l}$ phosphate-buffered saline (PBS, $\mathrm{pH}$ 7.4) only and did not receive EA stimulation.

Measurements of thermal hyperalgesia. Rats were immediately placed individually on a heated $\left(56^{\circ} \mathrm{C}\right)$ platform and the latency of the first sign of paw licking or jumping to avoid heat pain was recorded by an automatic electronic timer (Harvard Apparatus, Holliston, MA, USA). A cut-off period of $20 \mathrm{~s}$ was used. The latency response was monitored for 5 days following CFA injection, with or without EA stimulation.

qPCR. SYBR-Green PCR amplification of the whole L4-5 segments of the spinal cord was performed using an ABI PRISM 7900 sequence detection system (Applied Biosystems, Foster City, CA, USA). Reaction mixtures contained $10 \mathrm{pmol} / \mu \mathrm{l}$ of each primer and $2 \mathrm{X} \mathrm{SYBR}{ }^{\circledR}$ Green PCR Master mix (Applied Biosystems), which included HotStarTaqt DNA-polymerase in an optimized buffer, dNTP mix (with added dUTP), SYBR ${ }^{\circledR}$ Green I fluorescent dye, and ROX dye as a passive reference. Each of the 384-well, qPCR plates included serial dilutions $(1,1 / 2$ and $1 / 4,1 / 8$, and $1 / 16$ ) of cDNA, which were used to generate relative standard curves for the genes. Primers were amplified under the same conditions. The thermal cycling conditions were as follows: $50^{\circ} \mathrm{C}$ for $2 \mathrm{~min}$ and $95^{\circ} \mathrm{C}$ for $10 \mathrm{~min}$, followed by 40 cycles of $95^{\circ} \mathrm{C}$ for $30 \mathrm{sec}, 60^{\circ} \mathrm{C}$ for $30 \mathrm{sec}$ and $72^{\circ} \mathrm{C}$ for $30 \mathrm{sec}$. The qPCR cycle numbers were converted to gene amounts (ng). The primer sequences used were: Forward: 5'-GACAACTCAAGCGTCCAGAA-3' and reverse: 5'-CGTCGCTGACAATCTCAAGT-3' for GluR1; forward: 5'-GAGGACTACCGCAGAAGGAG-3' and reverse: 5'-GATCCTTTAGGTGTGGCGAT-3' for GluR2; forward: 5'-GTCTCCTGTGACTTCAACAG-3' and reverse: 5'-AGTTGTCATTGAGAGCAATGC-3' for glyceraldehyde 3-phosphate dehydrogenase (GAPDH).

Western blot analysis. To examine the changes in the AMPA subunits, the L4-5 segments of the spinal cord were removed following the termination of EA stimulation on day 5 of the experiment, under $10 \%$ chloral hydrate anesthesia $(350 \mathrm{mg} /$ $\mathrm{kg}$ injected intraperitoneally). The dorsal region of the spinal cord was excised from the ventral region and eventually the ipsilateral and contralateral regions of the injected side were obtained. The spinal cords were washed in cold HEPES buffer, and homogenized in nine volumes of lysis buffer. Equal quantities of protein were separated by $8-12 \%$ sodium dodecyl sulfate-polyacrylamide gel electrophoresis (SDS-PAGE). The resolved proteins were transferred to a nitrocellulose membrane (Whatman, Dassel, Germany), and the membrane was blocked with $5 \%$ non-fat milk in Trisbuffered saline containing $0.4 \%$ Tween-20.

The membranes were incubated with anti-GluR1 (Abcam, Cambridge, UK), anti-phospho-GluR1 (pGluR1, ser845; Millipore, Billerica, MA, USA), anti-GluR2 (Abcam) and anti-phosho-GluR2 (pGluR2, ser880; Abcam) for 1-2 h at room temperature. The blots were incubated with horseradish peroxidase-conjugated secondary antibody and the antibody-specific proteins were visualized by the enhanced chemiluminescence detection system (Pierce, Rockford, IL, USA). $\beta$-actin was used as a loading control for all experiments. Quantification of the immunoreactivity corresponding to the total and phosphorylated bands was performed by densitometric analysis using Multi Gauge software, version 3.0 (Fujifilm, Tokyo, Japan).

Immunohistochemistry. Rats anesthetized with $10 \%$ chloral hydrate were intracardially perfused with saline and $4 \%$ paraformaldehyde in PBS. The L4-5 segments of the spinal cord were removed, post-fixed in $4 \%$ paraformaldehyde for $4 \mathrm{~h}$ at $4^{\circ} \mathrm{C}$, and immersed in $30 \%$ sucrose for $48 \mathrm{~h}$ at $4^{\circ} \mathrm{C}$ for cryoprotection. Frozen $30 \mu \mathrm{m}$ sections were prepared and collected in $0.1 \mathrm{M}$ phosphate buffer ( $\mathrm{PB}, \mathrm{pH}$ 7.4) to be processed immunohistochemically as free-floating sections. The sections were pre-incubated in $0.3 \%$ hydrogen peroxide for $15 \mathrm{~min}$, rinsed thoroughly, and then incubated in a blocking solution containing 3\% normal goat serum and $0.3 \%$ Triton $\mathrm{X}-100$ in PBS for $30 \mathrm{~min}$ at room temperature. Sections were incubated for $16 \mathrm{~h}$ at $4^{\circ} \mathrm{C}$ with the same primary antibody used in the western blot analysis diluted in PBS containing $0.3 \%$ Triton X-100. Sections were washed with PBS, incubated with biotinylated anti-rabbit IgG secondary antibody (Vector, Burlingame, CA, USA) for $30 \mathrm{~min}$ and then washed again with PBS.

Sections were further incubated with an avidin-biotin-peroxidase complex kit (Vector) for $60 \mathrm{~min}$ at room temperature and peroxidase activity was detected using the DAB Peroxidase Substrate kit (Vector). To quantify the laminar expression of AMPA, the ipsilateral dorsal horn of the spinal cord was divided into three regions: The superficial dorsal horn (SDH, laminae I and II), the nucleus proprius (NP, laminae III and IV), and the neck of the dorsal horn (NECK, laminae V and VI). To visualize the expression of the AMPA receptor subunits, images of the dorsal horns were captured at x100 magnification using an AxioCam digital CCD camera (Zeiss, Jena, Germany). The integrated optical density (IOD) of each region of the dorsal horn was measured automatically using Visus Image Analysis software (Foresthill Products, Foresthill, CA, USA).

Statistical analysis. Data are expressed as the mean \pm SEM, and a Student's t-test was performed using the SigmaPlot software (SyStat Software, San Jose, CA, USA). P<0.05 was considered to indicate a statistically significant difference. 


\section{Results}

Thermal thresholds. Hyperalgesia was examined using the measurements of hot plate latency at 1-day intervals following CFA injection. Among the 10 rats in each group, only five rats demonstrating significant EA analgesia were selected and included in the behavioral analysis (Fig. 1). The hot plate latency of the ipsilateral hindpaw of CFA-treated rats was significantly decreased compared with that of the control rats. There was no significant difference in latency in pain resulting from noxious thermal stimuli, 1 and 2 days following CFA injection, between the CFA-treated rats with and without EA stimulation. However, EA stimulation significantly inhibited hyperalgesia induced by CFA from day 3 onwards.

qPCR analysis of AMPA receptor subunits. To determine whether the effects of EA on CFA-induced hyperalgesia were associated with AMPA receptor subunit mRNA, the expression of AMPA receptor GluR1 and GluR2 subunits was determined by qPCR in the L4-5 segments. Five rats presenting with significant EA analgesia 5 days following CFA injection were used for the mRNA analysis. There were no significant changes in the GluR1 mRNA in response to CFA injection with or without EA stimulation. However, GluR2 mRNA was significantly decreased in these groups (CFA treated with or without EA stimulation) (Fig. 2).

Western blot analysis of the AMPA receptor subunits. For the western blot analysis, the ipsilateral and contralateral dorsal region of the L4-5 segments of the spinal cord were removed at day 5 of the experiment, and the expression of the total and the phosphorylated GluR1 and GluR2 subunits was determined. The protein levels of each subunit were normalized to that of $\beta$-actin in the same sample. The increase of activity in the AMPA subunits was calculated as the ratio of phosphorylated subunits to total subunits and compared with that in the ipsilateral region of control rats. Phosphorylation of the GluR1 subunit was only marginally changed and not significantly different in CFA-injected rats with and without EA stimulation, compared with the control rats (Fig. 3A). However, the phosphorylation of GluR2 was significantly increased in CFA-injected rats in the ipsilateral dorsal horn of the spinal cord. The increased pGluR2 level was blocked by EA treatment (Fig. 3B).

Immunohistochemical analysis of the AMPA receptor subunits. The ipsilateral GluR1 and GluR2 subunits are widely distributed in the SDH region of the spinal cord. The expression of the GluR1 and GluR2 subunits did not exhibit significant changes following CFA injection with or without EA stimulation (data not shown). The expression of the phosphorylated GluR1 and GluR2 subunits was increased in the $\mathrm{SDH}$ region of CFA-injected rats compared with the control rats, and was significantly inhibited by EA stimulation (Fig. 4).

\section{Discussion}

EA is modified from traditional acupuncture, as the manual stimulations of the needle are replaced by electric pulse generation. EA treatment is widely used in the clinic to relieve

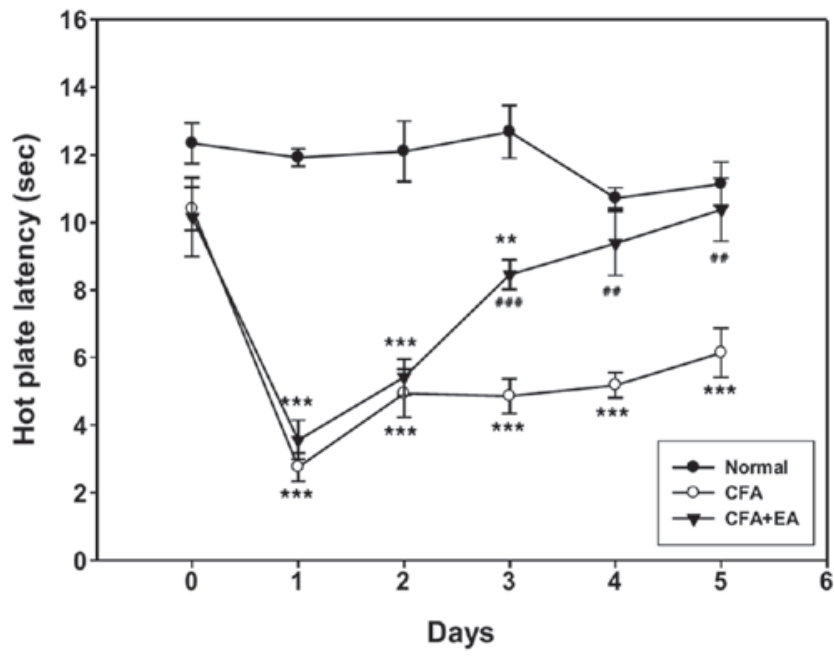

Figure 1. Analgesic effects of electroacupuncture (EA) in rats injected with complete Freund's adjuvant (CFA). Each point indicates the mean \pm SEM $(n=5)$. EA treatment led to significant inhibition of CFA-induced hyperalgesia from day 3 post-injection. ${ }^{* *} \mathrm{P}<0.01$ and ${ }^{* * * *} \mathrm{P}<0.001$ compared with the normal rats. ${ }^{\#} \mathrm{P}<0.01$ and ${ }^{\# \#} \mathrm{P}<0.001$ compared with the CFA-injected rats.

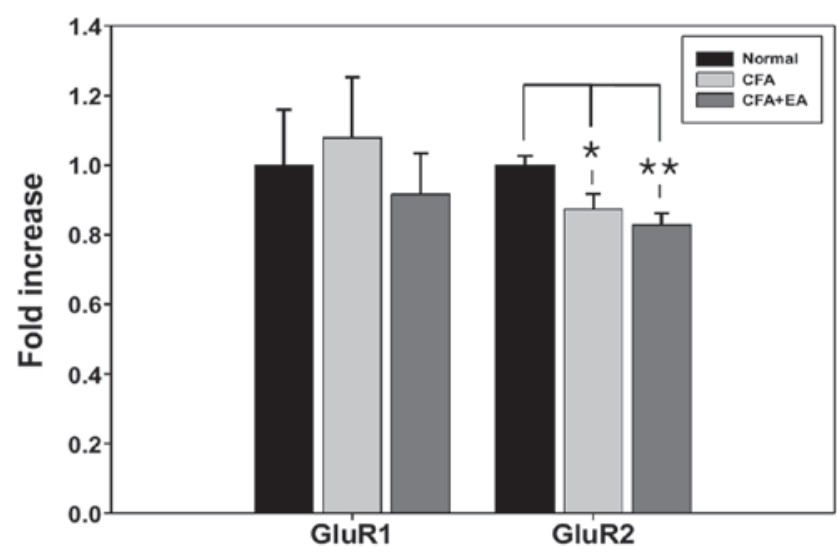

Figure 2. Effects of electroacupuncture (EA) stimulation on the expression of GluR1 and GluR2 mRNA in the L4-5 segments of the spinal cord $(n=5)$. A significant decrease in GluR2 mRNA in the rats injected with complete Freund's adjuvant (CFA) (with or without EA stimulation) compared with the normal rats is demonstrated. ${ }^{*} \mathrm{P}<0.05$ and ${ }^{* *} \mathrm{P}<0.01$.

acute or chronic pain in human patients, and may contribute to prolonged analgesia lasting for hours or days (11). The long-term synaptic alterations between primary afferent fibers and neurons in the spinal dorsal horn involve the activation of ionotropic glutamate receptors (12). The AMPA receptor in the spinal dorsal horn may be involved in EA analgesia (10). However, the exact involvement of this receptor in chronic pain remains to be elucidated.

The AMPA receptor is composed of the GluR1, GluR2, GluR3 and GluR4 subunits. GluR1 and GluR2 are predominant in the superficial laminae of the spinal dorsal horn (5). Therefore, it was hypothesized that these AMPA receptor subunits may be involved in the control of inflammatory nociception by EA stimulation through their expression and phosphorylation. Thus, EA stimulation at $2 \mathrm{~Hz}$ with $1 \mathrm{~mA}$ intensity in the CFA-induced pain rat model was used to select rats demonstrating significant EA analgesia. 
A
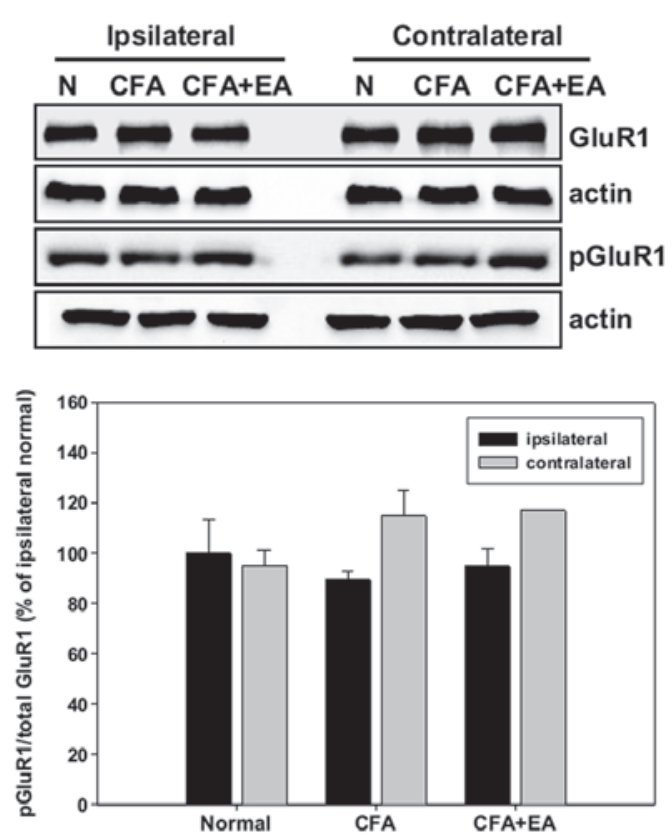

B
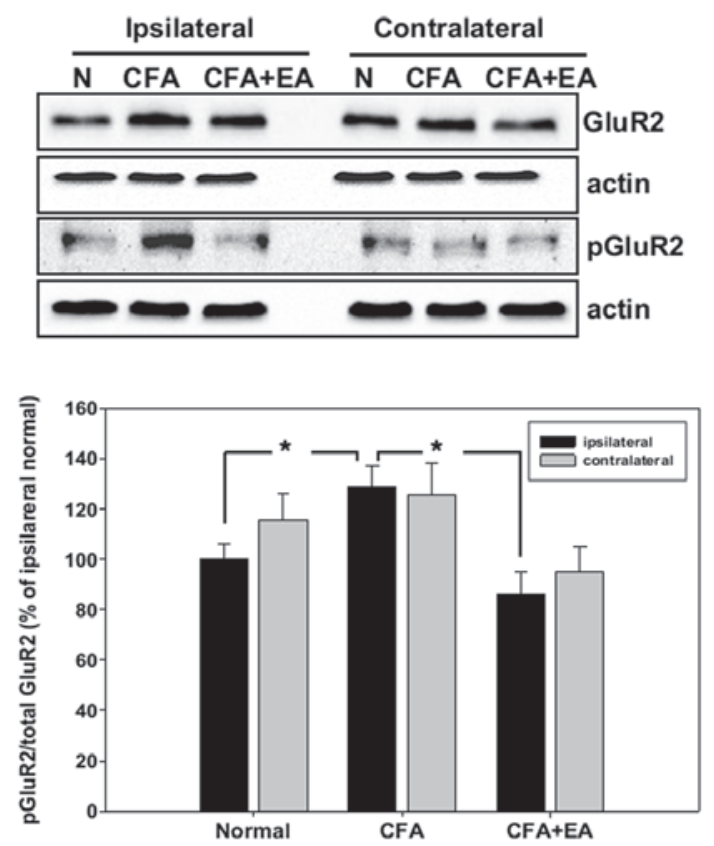

Figure 3. Western blotting and its densitometric analysis for the (A) GluR1 and (B) GluR2 subunits in the dorsal part of the L4-5 segments of the spinal cord. Levels for each protein are expressed as a percentage of that of the ipsilateral part of control rats. Each experimental result was obtained from five pooled animals. The panel represents a typical result from three independent experiments. There is a marked increase in phospho-GluR2 (pGluR2) of the ipsilateral dorsal part in the rats injected with complete Freund's adjuvant (CFA) compared with that in the normal rats, and this expression is inhibited by electroaccupuncture (EA) stimulation. ${ }^{*} \mathrm{P}<0.05$.

$\mathbf{A}$
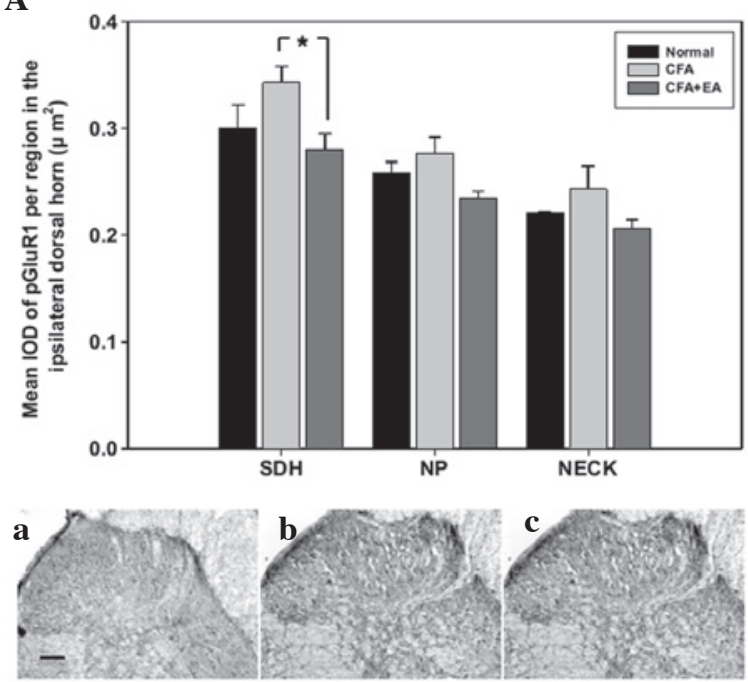

B
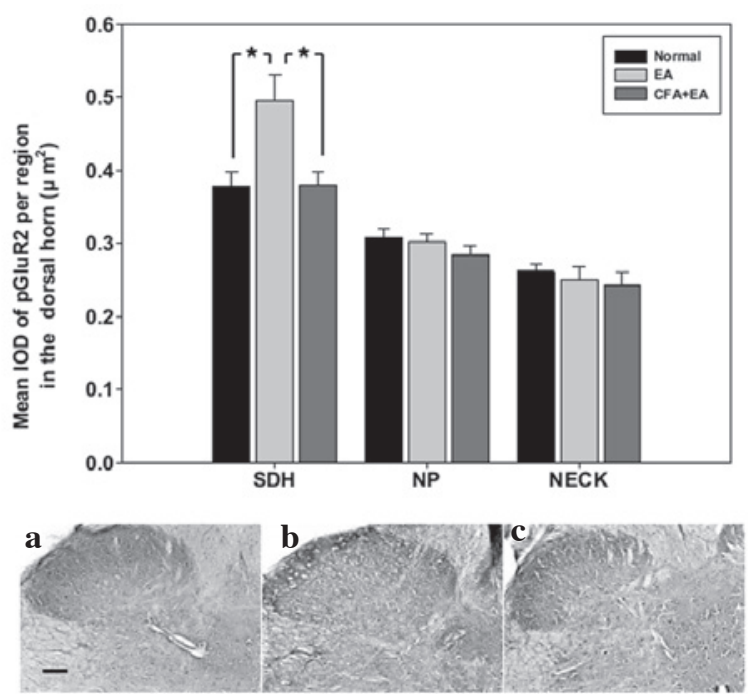

Figure 4. Immunohistochemistry and its densitometric analysis for the (A) phospho-GluR1 (pGluR1) and (B) pGluR2 subunits in the ipsilateral dorsal horn of the L4-5 segments $(n=5)$. Data are expressed as the mean \pm SEM. There is a marked increase in the mean integrated optical density (IOD) of pGluR2 in superficial dorsal horn (SDH) regions in the rats treated with complete Freund's adjuvant (CFA) compared with the controls, and this increase is inhibited by electroacupuncture (EA) stimulation. (a) normal rats; (b) CFA-treated rats; (c) CFA with EA-treated rats. Scale bar, $200 \mu \mathrm{m}$. "P<0.05.

CFA treatment induced thermal hyperalgesia lasting up to 5 days following injection and the significant inhibition of hyperalgesia by EA stimulation was evident from day 3 following injection (Fig. 1). The GluR1 and GluR2 subunit mRNAs of the AMPA receptor were examined by qPCR at day 5 following CFA injection. A previous study demonstrated a significant upregulation of the mRNAs following CFA injection and a return to the control levels by day 7 (GluR1-flop) and day 3 (GluR2-flip) (5). Furthermore, another study demonstrated a $25 \%$ bilateral decrease in GluR 1 expression in the substantia gelatinosa at the level of the lumbar cord 1 day following injection with lipopolysaccharide, with no significant change in the GluR2 levels (13).

These up- and downregulations correlate with the early development of hyperalgesia following inflammation. A previous study demonstrated specific regulation of the 
AMPAR subunits in response to the development of inflammatory hyperalgesia $(5,13)$ and during the sampling period after inflammation (14). In the present study, the level of GluR2 subunit mRNA in CFA-treated rats was significantly decreased compared with that in the control rats (Fig. 2), suggesting that the selective expression of the GluR2 subunit is related to the maintenance of chronic pain or the sampling period.

Increased GluR1 and GluR2 expression has been detected early after inflammation (5), but CFA injection does not alter the expression of GluR1 and GluR2 proteins in rats $(8,9)$. GluR1 expression is marginally increased after CFA injection, but the change is not significant and the GluR2 levels are not affected (15). The expression of the GluR1 and GluR2 subunits predominantly occurs in the superficial laminae of the spinal dorsal horn $(13,16)$.

AMPA receptor phosphorylation of serine residues is a mechanism for modulating channel properties in the central nervous system $(2,8)$. Upregulation of the phosphorylated GluR1 serine residues has been demonstrated in the dorsal horn following capsaicin or CFA treatment $(8,17)$. These results indicate that the phosphorylation of the GluR1 subunit participates in the induction of inflammatory pain.

The expression of each protein in the CFA-injected rats as a percentage of such expression in the control rats in the western blot analyses demonstrated a change in the phosphorylation of the GluR1 subunit between the control rats and the CFA-injected rats, with or without EA stimulation. However, these changes were not statistically significant (Fig. 3A). By contrast, the phosphorylation of GluR2 was significantly increased by CFA injection and this induction was inhibited by EA stimulation (Fig. 3B). Furthermore, the regional distribution of the subunits was examined by immunohistochemistry. The expression of the GluR2 subunit was not significantly altered at day 5 following CFA injection (data not shown). The phosphorylated form of GluR2 was significantly increased by the injection of CFA and inhibited by EA stimulation in the SDH region (Fig. 4B).

CFA-induced inflammation activated spinal protein kinase $\mathrm{C}$ and induced the phosphorylation of the serine 880 residue of GluR2 in the dorsal horn, which promotes the internalization of GluR2. The internalization of the GluR2 subunit of the AMPA receptor leads to the maintenance of CFA-induced pain hypersensitivity by increasing the permeability of the membrane to calcium (6).

The present data suggest that the phosphorylation of serine 880 of the GluR2 subunit is increased in the spinal cord following CFA injection. This increase in phosphorylated GluR2 may be significantly blocked by peripheral EA stimulation. A previous study demonstrated that CFA-induced peripheral inflammation leads to GluR2 internalization and GluR1 membrane insertion in the dorsal horn neurons, without altering the expression and distribution of the total GluR1 and GluR2 proteins in the dorsal horn (9).

The involvement of the spinal AMPA receptor in EA analgesia remains to be elucidated. The anti-pGluR2 used in the present study detected phosphorylation at serine 880 and greater changes in the GluR2 subunit upon EA stimulation. The majority of AMPA receptors of principal neurons contain the GluR2 subunit, rendering them impermeable to calcium. However, certain AMPA receptors lacking this subunit are expressed under pathological conditions (3). In addition, AMPA receptors undergo selective transcriptional and translational modulation following prolonged inflammation (14).

In summary, the present results suggest that the AMPA receptor may be involved in EA analgesia for chronic inflammatory pain by regulating the expression and phosphorylation state of its subunits. The GluR2 subunit of the AMPA receptor may be important in EA analgesia through its modulation of the phosphorylation state.

\section{References}

1. Collingridge GL, Isaac JT and Wang YT: Receptor trafficking and synaptic plasticity. Nat Rev Neurosci 5: 952-962, 2004.

2. Gao X, Kim HK, Chung JM and Chung K: Enhancement of NMDA receptor phosphorylation of the spinal dorsal horn and nucleus gracilis neurons in neuropathic rats. Pain 116: 62-72, 2005.

3. Katano T, Furue H, Okuda-Ashitaka E, et al: N-ethylmaleimidesensitive fusion protein (NSF) is involved in central sensitization in the spinal cord through GluR2 subunit composition switch after inflammation. Eur J Neurosci 27: 3161-3170, 2008.

4. Burnashev N, Khodorova A, Jonas P, et al: Calcium-permeable AMPA-kainate receptors in fusiform cerebellar glial cells. Science 256: 1566-1570, 1992.

5. Zhou QQ, Imbe H, Zou S, Dubner R and Ren K: Selective upregulation of the flip-flop splice variants of AMPA receptor subunits in the rat spinal cord after hindpaw inflammation. Brain Res Mol Brain Res 88: 186-193, 2001.

6. Park JS, Voitenko N, Petralia RS, et al: Persistent inflammation induces GluR2 internalization via NMDA receptor-triggered PKC activation in dorsal horn neurons. J Neurosci 29: 3206-3219, 2009.

7. Kerr RC, Maxwell DJ and Todd AJ: GluR1 and GluR2/3 subunits of the AMPA-type glutamate receptor are associated with particular types of neurone in laminae I-III of the spinal dorsal horn of the rat. Eur J Neurosci 10: 324-333, 1998.

8. Lu Y, Sun YN, Wu X, et al: Role of alpha-amino-3-hydroxy-5methyl-4-isoxazolepropionate (AMPA) receptor subunit GluR1 in spinal dorsal horn in inflammatory nociception and neuropathic nociception in rat. Brain Res 1200: 19-26, 2008.

9. Park JS, Yaster M, Guan X, et al: Role of spinal cord alphaamino-3-hydroxy-5-methyl-4-isoxazolepropionic acid receptors in complete Freund's adjuvant-induced inflammatory pain. Mol Pain 4: 67, 2008.

10. Choi BT, Lee JH, Wan Y and Han JS: Involvement of ionotropic glutamate receptors in low frequency electroacupuncture analgesia in rats. Neurosci Lett 377: 185-188, 2005.

11. Melzack R, Vetere P and Finch L: Transcutaneous electrical nerve stimulation for low back pain. A comparison of TENS and massage for pain and range of motion. Phys Ther 63: 489-493, 1983.

12. Chen J and Sandkühler J: Induction of homosynaptic long-term depression at spinal synapses of sensory a delta-fibres requires activation of metabotropic glutamate receptors. Neuroscience 98: 141-148, 2000.

13. Pellegrini-Giampietro DE, Fan S, Ault B, Miller BE and Zukin RS: Glutamate receptor gene expression in spinal cord of arthritic rats. J Neurosci 14: 1576-1583, 1994.

14. Guan Y, Guo W, Zou SP, Dubner R and Ren K: Inflammationinduced upregulation of AMPA receptor subunit expression in brain stem pain modulatory circuitry. Pain 104: 401-413, 2003.

15. Lee J and Ro JY: Differential regulation of glutamate receptors in trigeminal ganglia following masseter inflammation. Neurosci Lett 421: 91-95, 2007.

16. Garry EM and Fleetwood-Walker SM: A new view on how AMPA receptors and their interacting proteins mediate neuropathic pain. Pain 109: 210-213, 2004.

17. Nagy GG, Al-Ayyan M, Andrew D, Fukaya M, Watanabe M and Todd AJ: Widespread expression of the AMPA receptor GluR2 subunit at glutamatergic synapses in the rat spinal cord and phosphorylation of GluR1 in response to noxious stimulation revealed with an antigen-unmasking method. J Neurosci 24: 5766-5777, 2004. 